\title{
Images of the month: Pulmonary cement embolism
}

\author{
Authors: Chan Sin Chai, ${ }^{\mathrm{A}}$ Sze Shyang Kho, ${ }^{\mathrm{A}}$ Swee Kim Chan, ${ }^{\mathrm{A}}$ Teng Teng Tee ${ }^{\mathrm{A}}$ and Siew Teck Tie ${ }^{\mathrm{B}}$
}

Percutaneous vertebroplasty (PV) involves injection of polymethylmethacrylate bone cement into vertebral body for relief of pain and strengthening of bone in symptomatic vertebral compression fractures.

Passage of bone cement into vertebral venous plexus and then into the lungs is a rare and serious complication of PV. The reported incidence up to $26 \%$.

We present an incidental finding of pulmonary cement embolism (PCE) after PV. A 68-year-old woman with history of PV 3 years previously for T11 osteoporotic fracture presented to us with cough for 3 weeks following choking on a fish bone.

Chest X-ray showed left lower zone consolidation and a high-density opacity in a tubular branching pattern, corresponding to pulmonary arterial distribution. Contrasted computed tomography of the thorax showed segmental pulmonary cement embolism of both lungs and left lower lobe consolidation.

She underwent bronchoscopy with findings of a purulent secretion from the left lower lobe. Her symptoms resolved after 2 weeks of antibiotics. She was managed conservatively for the PCE as she remained asymptomatic.

This case highlights the need for a standard post-PV chest $X$-ray, as patients with cement embolisms can be completely asymptomatic. Measures to minimise the risk of pulmonary cement embolisms during PV need to be taken.

KEYWORDS: percutaneous vertebroplasty, pulmonary cement embolism, osteoporotic fracture

DOI: 10.7861/clinmed.2020-0913

\section{Case presentation}

A 68-year-old woman presented to us with cough for 3 weeks following choking on a fish bone. She was not dyspnoeic nor septic, with oxygen saturation of $98 \%$ on room air and total white cells of $10 \times 10^{9} / \mathrm{L}$. There were left lower zone crepitations on lung auscultation. Admission chest X-ray showed left lower zone consolidation, but surprisingly also revealed bilateral central highdensity tubular opacities in a branching pattern, corresponding to the pulmonary arterial distribution.

Authors: ${ }^{\text {A }}$ physician, Sarawak General Hospital, Kuching, Malaysia;

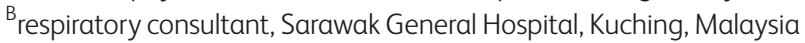

Upon further questioning, the patient revealed her history of percutaneous vertebroplasty (PV) 3 years previously for T11 osteoporotic fracture. We proceeded with computed tomography, which demonstrated segmental pulmonary cement embolism in both lungs with evidence of cement leakage from the previous PV site through the azygos and hemiazygos venous system and into the pulmonary arterial circulation, consistent with segmental pulmonary cement embolism in both lungs (Figs 1 and 2).

The patient subsequently underwent flexible bronchoscopy, which revealed purulent secretion only from the left lower lobe, with no foreign body, consistent with aspiration pneumonia. Her acute symptoms resolved after 2 weeks of antibiotics. She was managed conservatively for the central pulmonary cement embolism, as the event was years past, and she remained asymptomatic.

\section{Discussion}

PV involves injection of polymethylmethacrylate (PMMA) bone cement into the vertebral body for relief of pain and strengthening of bone in symptomatic vertebral compression fractures not feasible for definitive operation. Passage of bone cement into the vertebral venous plexus and then into the lungs is a rare but potentially serious complication of PV with the reported incidence up to $26 \%$.

Patients with cement embolism can present with a spectrum of symptoms - the diagnosis was picked up incidentally in our case when she presented with an unrelated aspiration pneumonia.

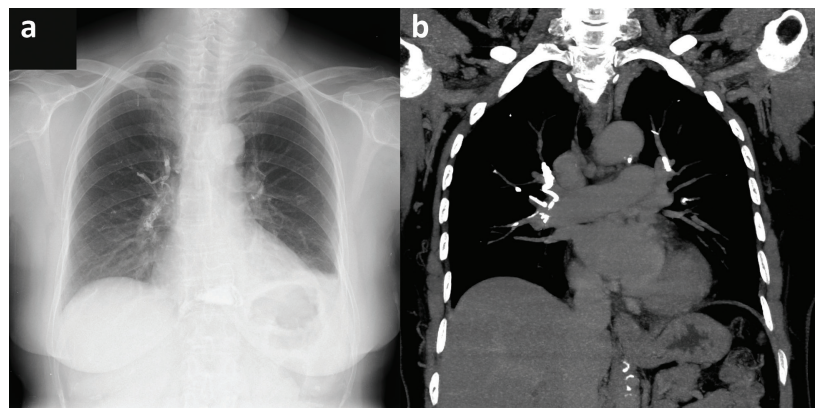

Fig 1. a) Plain chest X-ray showing high-density opacity in tubular branching pattern corresponding to pulmonary arterial distribution and left lower lobe consolidation. b) Coronal computed tomography of the thorax revealing segmental pulmonary cement embolism in both lungs. 


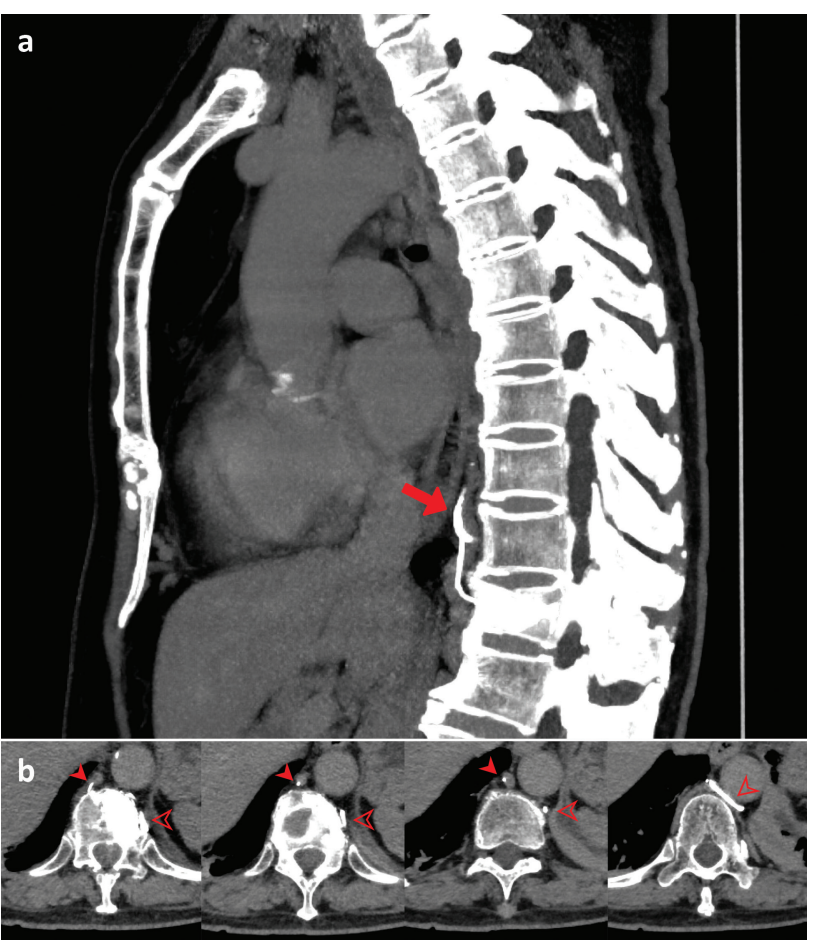

Fig 2. a) Sagittal computed tomography of the thorax demonstrates the cement leaking from T11 spine into the vertebral venous plexus (red arrow). b) Axial computed tomography showing evidence of cement leaking from PV site into the azygos (filled arrow) and hemiazygos (transparent arrow) venous system into pulmonary arterial circulation.

No treatment is recommended for asymptomatic patients with peripheral cement embolisms. Surgical treatment with embolectomy is suggested for symptomatic central embolisms. Whereas, for symptomatic peripheral embolisms, or asymptomatic central embolisms as in our case, initial treatment should have been heparinisation followed by 3-6 months of warfarin therapy. ${ }^{2}$

This case highlights the need for a standard post-PV chest $X$-ray, as patients with cement embolisms can be completely asymptomatic. Timely recognition of her central event would have prompted initiation of anticoagulation. Measures to minimise the risk of pulmonary cement embolisms include avoidance of prone positioning, utilisation of fluoroscopy or computed tomography monitoring during PV procedure, and usage of bone cement in appropriate amount and viscosity. ${ }^{3}$.

\section{Acknowledgements}

We thank our colleagues from Department of Diagnostic Imaging, Sarawak General Hospital, for a detailed analysis of the patient's medical imaging that greatly improved the manuscript.

\section{References}

1 Barbero S, Casorzo I, Durando M, et al. Percutaneous vertebroplasty: The follow-up. Radiol Med 2008;113:101-13.

2 Krueger A, Bliemel C, Zettl R et al. Management of pulmonary cement embolism after percutaneous vertebroplasty and kyphoplasty: A systematic review of the literature. Eur Spine J 2009;18:1257-65.

3 Baroud G, Crookshank M, Bohner M. High-viscosity cement significantly enhances uniformity of cement filling in vertebroplasty: an experimental model and study on cement leakage. Spine 2006;31: 2562-8.

Address for correspondence: Dr Chan Sin Chai, Respiratory Medicine Unit (RCU), Sarawak General Hospital, Jalan Hospital, 93586 Kuching, Sarawak, Malaysia.

Email: chansinchaics@gmail.com 